Bangladesh J. Plant Taxon. 16(1): 9-19, 2009 (June)

(C) 2009 Bangladesh Association of Plant Taxonomists

\title{
A TAXONOMIC ACCOUNT ON THE PHYTOPLANKTON OF A POND RECEIVING TEXTILE INDUSTRIAL EFFLUENTS. II. EUGLENOPHYCEAE AND BACILLARIOPHYCEAE
}

\author{
Z.N. TAHMIDA BEGUM ${ }^{1}$ \\ Department of Botany, University of Dhaka, Dhaka 1000, Bangladesh. \\ Keywords: Industrial effluents; Phytoplankton; Taxonomy; Euglenophyceae; Bacillariophyceae.
}

\begin{abstract}
A total of 97 phytoplanktonic algal taxa belonging to 17 genera under two classes (Euglenophyceae and Bacillariophyceae) have been recorded from a pond receiving effluents from two textile industries at Demra, Dhaka district. Five diatom species, namely Navicula cryptocephala Kütz., $N$. decussis Oestrup, N. rhynchocephala Kütz., $N$. viridula Kütz. and Nitzschia intermedia Hantzsch are new records for Bangladesh.
\end{abstract}

\section{Introduction}

For the assessment of water quality phytoplankton are important biological indicators and sometimes they are better than physical and chemical variables (Round, 1985). Previously, information about the physico-chemical aspects (Begum and Hossain, 1993), and impact of major and minor elements on the plankton community (Begum et al., 1996) of a pond receiving textile industrial effluents have been made for the first time from Bangladesh. Recently, Begum (2008) has described the phytoplanktonic algal taxa belonging to six classes, namely Cyanophyceae, Chlorophyceae, Xanthophyceae, Chrysophyceae, Cryptophyceae and Dinophyceae from the same pond at Demra, Dhaka district. The present paper is a continuation and last part of the previous one, based on the same collections, and it includes 97 taxa under Euglenophyceae and Bacillariophyceae.

\section{Materials and Methods}

The samples were collected from a pond receiving textile industrial effluents in different seasons of 1990 and 1991. Description of sample collections, their preservation and examination, details of physical and chemical conditions of water at four stations or sampling points have been published by Begum (2008). Literature consulted for the identification of all the phytoplankton are: Islam and Khatun (1966), Islam and Haroon (1975), Islam and Aziz (1977, 1979), Islam and Chowdhury (1979), Islam and Hossain (1979), Germain (1981), Islam and Moniruzzaman (1981), Aziz and Islam (1986), Islam and Mannan (1986), Khondker et al. (1990), Islam et al. (1991), Aziz and Ara (2000), Nahar (2001), Aziz and Tanbir (2003), Islam and Alfasane (2004), and Islam and Irfanullah (2005). In the present study classification proposed by Bold and Wynne (1985) is followed.

\footnotetext{
${ }^{1}$ E-mail: botany@univdhaka.edu
} 


\section{Taxonomic enumeration}

A total of 97 taxa belonging to 17 genera under two classes are presented in this paper, most of them have been reported previously from different aquatic bodies, mostly polluted water (see references in the Materials and Methods). However, five diatom taxa are newly recorded for Bangladesh as indicated after their brief descriptions.

\section{Class: Euglenophyceae}

\section{Order: Euglenales; Family: Euglenaceae}

1. Euglena acus (Müller) Ehr., Abhandl. königl. Akad. der Wiss. zu Berlin 1: 1-88, pl. 6 (1830). [Syn.: Vibrio acus Müller (1786), Euglena acus var. rigida Hübner (1886), E. acus var. minor Hansgirg (1892)]. Cells 76-250 $\mu \mathrm{m}$ long, 5.7-14.3 $\mu \mathrm{m}$ broad. Stations: 1-4; abundant.

2. Euglena australica var. claviformis Playfair, Proc. Linn. Soc. N. S. W. 48: 223 (1923). Cells $20 \mu \mathrm{m}$ long, $14 \mu \mathrm{m}$ broad. Stations: 1-3; common.

3. Euglena chlamydophora Mainx, Arch. f. Protistenk. 60: 342, pl. 1, fig. 8 (1928). Cells $54 \mu \mathrm{m}$ long, $17 \mu \mathrm{m}$ broad. Stations: 1-4; common.

4. Euglena clavata Skuja, Symb. Bot. Upsal. 9(3): 189, pl. 22, figs 2-5 (1948). Cells 99 $\mu \mathrm{m}$ long, $16 \mu \mathrm{m}$ broad. Stations: 2, 4; rare.

5. Euglena deses Ehr., Abhandl. d. Akad. d. Wiss. Berlin 1833: 248 (1834). [Syn.: Enchelys deses Müller (1786), Euglena satelles Braslavska-Spectorova (1937)]. Cells $99 \mu \mathrm{m}$ long, $11 \mu \mathrm{m}$ broad. Station: 2; rare.

6. Euglena exilis Gojdics, The Genus Euglena: 148, pl. 28, fig. 4 (1953). Cells $48 \mu \mathrm{m}$ long, $11.4 \mu \mathrm{m}$ broad. Stations: 1-3; few.

7. Euglena flava Dangeard, Le Botaniste 8: 180, pl. 5, figs. 53 (1902). Cells 16-44 $\mu \mathrm{m}$ long, 6.4-14.3 $\mu \mathrm{m}$ broad. Station: 3; rare.

8. Euglena geniculata Dujardin, Infusiones: 362 (1841). Cells $99 \mu \mathrm{m}$ long, $15.6 \mu \mathrm{m}$ broad. Stations: 1-4; common.

9. Euglena granulata (Klebs) Fr. Schmitz Jahrb. Wiss. Bot. 15: 16 (1884). [Syn.: E. velata var. granulata Klebs (1883)]. Cells 37-64 $\mu \mathrm{m}$ long, 23-29 $\mu \mathrm{m}$ broad. Stations: 1, 2, 4; few.

10. Euglena güntheri Gojdics, Univ. Wisconsin Press, Madison, p.161, fig.1, pl. 33 (1953). [Syn.: Enchelys terricola Günther (1928)]. Cells 54-97 $\mu \mathrm{m}$ long, 11.4-14.5 $\mu \mathrm{m}$ broad. Stations: 1, 2, 4; common.

11. Euglena pisciformis Klebs, Unters. bot. Inst. Tüb. 1(2): 302 (1883). [Syn.: E. agilis H. J. Carter (1856)]. Cells 24-82 $\mu \mathrm{m}$ long, 7-11 $\mu \mathrm{m}$ broad. Stations: 1-4; abundant. 
12. Euglena polymorpha Dangeard, Le Botaniste 8: 175 (1902). [Syn.: E. granulata var. polymorpha Popova (1966)]. Cells $73 \mu \mathrm{m}$ long, 6.6-23.8 $\mu \mathrm{m}$ broad. Stations: 1, 2; common.

13. Euglena proxima Dangeard, Le Botaniste 8: 154 (1902). Cells $54 \mu \mathrm{m}$ long, $14.2 \mu \mathrm{m}$ broad. Station: 3; rare.

14. Euglena sanguinea Ehr., Physik. Abh. kgl. Akad. Wiss. 1: 1-18, pl. 6.1830 (1831). [Syn.: Cercaria viridis Müller (1790), Euglena viridis var. sanguinea Playfair (1921)]. Cells 60-102 $\mu \mathrm{m}$ long, $31 \mu \mathrm{m}$ broad. Stations: 1-4; abundant.

15. Euglena sociabilis (Schimtz) Dangeard, La Botaniste 8: 182 (1902). Cells $70 \mu \mathrm{m}$ long, $7 \mu \mathrm{m}$ broad. Stations: 1-4; abundant.

16. Euglena spirogyra Ehr., Abh. K. Akad. Wiss. Berlin, Phys. K1. 1830: 83, pl. 6: 6 (1830). [Syn.: E. spirogyra var. fusiformis Deflandre (1924)]. Cells 70-74 $\mu \mathrm{m}$ long, 3-14 $\mu$ m broad. Stations: 1-4; common.

17. Euglena subehrenbergii Skuja, Symb. Bot. Upsal. 9(3): 192 (1948). Cells $92 \mu \mathrm{m}$ long, $11.5 \mu \mathrm{m}$ broad. Station: 1; rare.

18. Euglena tripteris (Dujardin) Klebs, Unters. Bot. Inst. Tüb. 1: 306 (1883). [Syn.: E. torta Stokes (1885), E. tripteris subsp. crassa Swirenko (1915)]. Cells 65-97 $\mu \mathrm{m}$ long, 11-17 $\mu \mathrm{m}$ broad. Station: 2; very rare.

19. Euglena variabilis Klebs, Unters. Bot. Inst. Tüb. 1: 300 (1883). Cells $77 \mu \mathrm{m}$ long, 14-29 $\mu \mathrm{m}$ broad. Stations: 1, 2; abundant.

20. Phacus caudatus Hübn., Euglenac.-F1. Stralsund: 5, fig. 5 (1886). Cells 18-30 $\mu \mathrm{m}$ long, $8.5-18.2 \mu \mathrm{m}$ broad. Stations: $1-3$; rare.

21. Phacus curvicauda Swirenko, Arch. f. Hydrobiol. v. Planktonk. 10: 333 pl. II, figs 13, 16 (1915). [Syn.: Phacus brevicauda (Klebs) Lemm. bei Fritsch (1918), P. brevicauda fa. minor Defl. (1928)]. Cells 30-80 $\mu \mathrm{m}$ long, 24-47 $\mu \mathrm{m}$ broad. Stations: $1-4$; abundant.

22. Phacus longicauda (Ehr.) Duj., Infusoires: 337 (1841). Cells $40 \mu \mathrm{m}$ long, $33 \mu \mathrm{m}$ broad. Stations: 1, 2, 4; few.

23. Phacus swirenkoi Skvortzov, Ber. d. Dtsch. Bot. Ges. 46 (105-125) (1928). Cells 38-70 $\mu \mathrm{m}$ long, 23-60 $\mu \mathrm{m}$ broad. Stations: 1, 3, 4; few.

24. Trachelomonas abrupta fa. angustata Defl., Monogr. Du genre Trachelomonas Nemurous (1926). Lorica 25-26 $\mu \mathrm{m}$ long, 14-18 $\mu \mathrm{m}$ broad. Stations: 1, 2; few.

25. Trachelomonas allorgei var. madaripurense Islam, Int. rev. der Gesamt. Hydrobiol. 66(1): 109-125 (1981). Lorica $71 \mu \mathrm{m}$ long, $18 \mu \mathrm{m}$ broad. Station: 1; rare.

26. Trachelomonas armata (Ehr.) Stein, Org. Infusionsthiere III(1): pl. 22, figs 37, 38 (1878). Lorica 12-14 $\mu \mathrm{m}$ long, 21-30 $\mu \mathrm{m}$ broad. Station: 1; rare. 
27. Trachelomonas armata var. steinii Lemm., Abh. Naturw. ver. Bremen 18: 165 (1905). Lorica 43-50 $\mu \mathrm{m}$ long, 31-39 $\mu \mathrm{m}$ broad. Stations: 2, 3; rare.

28. Trachelomonas caffra Rino, Revista de Ciências Biológicas [Universidade de Lourenco Marques], Série A, 5: 158, pl. 8, figs 6-9 (1972). Lorica with spines 32-33 $\mu \mathrm{m}$ long, $36 \mu \mathrm{m}$ broad; without spines $22-24 \mu \mathrm{m}$ long, 22-23 $\mu \mathrm{m}$ broad. Station: 2 ; rare.

29. Trachelomonas dybowskii Drez., Odbitka $z$ Rosprawi Widom. z Muz. im Dzieduszychich. 7/8 (1921). [Syn.: T. oblonga Lemm. bei Conrad und Van Meel (1952)]. Lorica 16-19 $\mu \mathrm{m}$ long, 9-17 $\mu \mathrm{m}$ broad. Station: 3; rare.

30. Trachelomonas hispida (Perty) Stein, Org. Infusionsthiere III(1): 22, figs 20-34 (1878). Lorica 21-36 $\mu \mathrm{m}$ long, 15-25 $\mu \mathrm{m}$ broad. Stations: 1-4; common.

31. Trachelomonas hispida var. coronata Lemm., Eugl. in Die Süssw. Deutsch. Öst. und der Schweiz, G. Fischer, Jena 2: 150 (1913). Lorica $38 \mu \mathrm{m}$ long, 22-25 $\mu \mathrm{m}$ broad. Stations: 2-4; few.

32. Trachelomonas hispida var. punctata Lemm., Abh. Naturw. ver. Bremen 18: 165 (1905). Lorica 25-29 $\mu \mathrm{m}$ long, 17-26 $\mu \mathrm{m}$ broad. Stations: 1-4; common.

33. Trachelomonas intermedia Dang., La Botaniste: 8 (97-360) (1901). Lorica 20-25 $\mu \mathrm{m}$ long, 18-21 $\mu \mathrm{m}$ broad. Station: 1 ; rare.

34. Trachelomonas mucosa var. brevicollis Skv., Ber. d. Dtsch. Bot. Ges. 43: 306-341 (1925). Lorica $18 \mu \mathrm{m}$ long, $14.5 \mu \mathrm{m}$ broad. Station: 1; rare.

35. Trachelomonas oblonga Lemm., Abh. Naturw. ver. Bremen 16: 344 (1899). Lorica 11-16 $\mu \mathrm{m}$ long, 7.5-12.4 $\mu \mathrm{m}$ broad. Stations: 1-4; common.

36. Trachelomonas oblonga var. attenuata Playfair, Proc. Linn. Soc: N. S. W., Sydney 40: 1-41 (1915). [Syn.: T. minuscula Drez. (1925)]. Lorica $16.4 \mu \mathrm{m}$ long, $12.3 \mu \mathrm{m}$ broad. Station: 2; rare.

37. Trachelomonas oblonga var. truncata Lemm., Beih. Bot. Zbl. 76: 150-156 (1898). Lorica 10-11 $\mu \mathrm{m}$ long, $7.5 \mu \mathrm{m}$ broad. Station: 2; rare.

38. Trachelomonas oblonga Lemm. fa. ovata Defl., Monogr. Du genre Trachelomonas -Nemurous (1926). Lorica $17.7 \mu \mathrm{m}$ long, $13.6 \mu \mathrm{m}$ broad. Station: 1; rare.

39. Trachelomonas planctonica Swir., Arch. Hydrobiol. Planktonk. 9: 633 (1914). Lorica 26-29 $\mu \mathrm{m}$ long, $21 \mu \mathrm{m}$ broad. Station: 2; rare.

40. Trachelomonas playfairii Defl., Bull. Soc. Bot. France 71: 1125 (1924). [Syn.: T. flexicollis Drez. (1925), non T. flexicollis Drez. (1922)]. Lorica 23-25 $\mu \mathrm{m}$ long, 16-18 $\mu \mathrm{m}$ broad. Stations: 1-4; few.

41. Trachelomonas pulcherrima Playfair, Proc. Linn. Soc.: N. S. W., Sydney 40: 13 (1915). [Syn.: T. oblonga var. pulcherrima (Playfair) Popova (1955)]. Lorica 20.0$21.5 \mu \mathrm{m}$ long, $10.0-11.5 \mu \mathrm{m}$ broad. Station: 2 ; rare. 
42. Trachelomonas pulcherrima var. latior Playfair, Proc. Linn. Soc.: N. S. W., Sydney 40: 1-41 (1915). Lorica $20 \mu \mathrm{m}$ long, $11 \mu \mathrm{m}$ broad. Station: 2; vary rare.

43. Trachelomonas pulcherrima var. ovalis Playfair, Proc. Linn. Soc.: N. S. W., Sydney 40: 14 (1915). [Syn.: T. oblonga var. ovalis Popova (1955)]. Lorica $19.6 \mu \mathrm{m}$ long, 10.0-11.4 $\mu \mathrm{m}$ broad. Station: 2 ; rare.

44. Trachelomonas pusilla Playfair, Proc. Linn. Soc.: N. S. W., Sydney 40: 12 (1915). Lorica 12.4-14.3 $\mu \mathrm{m}$ long, 10.7-12.4 broad. Stations: 1-3; common.

45. Trachelomonas robusta Swir. emend Defl., Arch. Hydrobiol. 9: 636 (1914). Lorica 28.0-28.6 $\mu \mathrm{m}$ long, 21.5-22.0 $\mu \mathrm{m}$ broad. Station: 2 ; rare.

46. Trachelomonas rotunda Swir., Arch. Hydrobiol. 9: 636 (1914). [Syn.: T. gibbelaris var. rotunda Skv. (1925)]. Lorica 12.0-22.5 $\mu \mathrm{m}$ long, 14-25 $\mu \mathrm{m}$ broad. Stations: 1-4; abundant.

47. Trachelomonas superba Swir., Arch. Hydrobiol. 9: 642 (1914). [Syn.: T. horrida var. superba (Swirenk.) Skv. (1917)]. Lorica 31-41 $\mu \mathrm{m}$ long, 25-35 $\mu \mathrm{m}$ broad. Stations: 2, 4; few.

48. Trachelomonas volvocina Ehr., Phys. Abh. Berl. Akad. Wiss: 145-336 (1833). Lorica 7.0-21.5 $\mu \mathrm{m}$ in diameter. Stations: 1-4; abundant.

49. Trachelomonas volzii Lemm., Arch. Hydrobiol. 1: 409-427 (1906). Lorica 31-32 $\mu \mathrm{m}$ long, 16.5-18.0 $\mu \mathrm{m}$ broad. Stations: 1, 2, 4; few.

50. Trachelomonas volzii Lemm. var. acidophila Bourr., Bull. Inst. Franç. Afr. Noire, ser. A, 23: 310, 354, pl. 5, fig. 13, pl. 6, fig. 2. (1961). Lorica 36-37 $\mu \mathrm{m}$ long, 14-16 $\mu \mathrm{m}$ broad. Station: 1; rare.

\section{Class: Bacillariophyceae}

\section{Order: Centrales; Family: Melosiraceae}

51. Melosira granulata (Ehr.) Ralfs, Pritchard, Hist. Inf. ed. 4: 820 (1861). [Syn.: $M$. moniliformis Ag. (1824)]. Frustules 11.5-15.5 $\mu \mathrm{m}$ long, $8.0 \mu \mathrm{m}$ broad; half-cells 3.6 $\mu \mathrm{m}$ long. Stations: 1-4; abundant.

52. Melosira varians C. Ag., Flora 10(40): 628 (1827). [Syn.: Lysigonium varians (C. Agardh) De Tone (1892)]. Frustules 20-22 $\mu \mathrm{m}$ long, $9.5 \mu \mathrm{m}$ broad, spine $7.3 \mu \mathrm{m}$ long. Stations: 1-3; few.

\section{Family: Coscinodiscaceae}

53. Coscinodiscus lacustris Grun., K. Svenska Vet.-Akad. Handl., ser. 4, 17(2): 114 (1880). Frustules 33-34 $\mu \mathrm{m}$ in diameter in valve view. Stations: 1-3; few. 


\section{Order: Pennales; Family: Fragilariaceae}

54. Fragilaria capucina Desm., Pl. Crypt. France ed. 1, 10: 453 (1825). [Syn.: F. capucina var. lanceolata Grunow (1881)]. Frustules $44 \mu \mathrm{m}$ long, $4.5 \mu \mathrm{m}$ broad. Stations: 2-4; common.

55. Fragilaria virescens Ralfs, Ann. Mag. Nat. Hist. 12: 110 (1843). Frustules $42-79 \mu \mathrm{m}$ long, 5-8 $\mu \mathrm{m}$ broad, striae 9-12 in $10 \mu \mathrm{m}$. Stations: 1-4; common.

56. Synedra acus Kütz., Kieselschal. Bacill: 68, pl. 15, fig. 7 (1844). Frustules $149 \mu \mathrm{m}$ long, $73 \mu \mathrm{m}$ broad (at the middle), $3.6 \mu \mathrm{m}$ broad (at the tip), striae 14-16 in $10 \mu \mathrm{m}$. Stations: $1-4$; abundant.

57. Synedra tabulata (Ag.) Kütz., Bacillarien p. 68 (1844). [Syn.: Diatoma tabulatum Ag. (1832)]. Frustules 33-94 $\mu \mathrm{m}$ long, 4.0-5.5 $\mu \mathrm{m}$ broad, striae 12 in $10 \mu \mathrm{m}$. Stations: $1-4$; abundant.

58. Synedra ulna (Nitzch) Ehr., Abh. K. Akad. Wiss. Berlin 1831, Phys. K1. 87 (1932). [Syn: Bacillaria ulna Nitzsch.]. Frustules 320-350 $\mu \mathrm{m}$ long, $6.6 \mu \mathrm{m}$ broad in the median region; tip $9.5 \mu \mathrm{m}$ broad. Stations: 1-4; abundant.

59. Synedra ulna var. oxyrhynchus (Kütz.) Ơ Meara, Proc. Ray. Trish Acad., ser. 2 (science): 306 (1875). Frustules 120-126 $\mu \mathrm{m}$ long, $14 \mu \mathrm{m}$ broad, 10 or 11 striae in 10 $\mu \mathrm{m}$. Stations: 1-4; common.

\section{Family: Eunotiaceae}

60. Eunotia alpina (Näg.) Hust., A. Schmidt's Atlas: pl. 291, figs 7, 8 (1913). [Syn.: Synedra alpina Nägeli (1849)]. Frustules 57-100 $\mu \mathrm{m}$ long, 2.5-3.5 $\mu \mathrm{m}$ broad in the median region. Stations: 2-4; common.

61. Eunotia lunaris (Ehr.) Grun., Month. Micr. J. 18: 170 (1877). Frustules $112 \mu \mathrm{m}$ long, $73 \mu \mathrm{m}$ broad (at the middle), 15 striae in $10 \mu \mathrm{m}$. Stations: 1-4; abundant.

62. Eunotia monodon Ehr., Ath. K. Akad. Wiss. Berlin Physik. Kl. 1841: 414 (1843). [Syn.: E. undoso var. monodon Ehr. (1870), E. major (W. Smith) Rabenhorst (1864)]. Frustules $54 \mu \mathrm{m}$ long, 7-26 $\mu \mathrm{m}$ broad (at the middle), 9-12 striae in $10 \mu \mathrm{m}$. Stations: 1, 2, 4; few.

63. Eunotia pectinalis var. minor (Kütz.) Rab., Bot. Jahrb. 45 : 117 (1910). Frustules $43.5 \mu \mathrm{m}$ long, $3.6 \mu \mathrm{m}$ broad (at the middle), 14 or 15 striae in $10 \mu \mathrm{m}$. Stations: 2,4 ; very rare.

64. Eunotia sudetica (O. Müller) Forsch, Ber. Biol. Stat. Plön 6: 59 (1898). Frustules 33 $\mu \mathrm{m}$ long, $15 \mu \mathrm{m}$ broad, 9 or 10 striae in $10 \mu \mathrm{m}$. Stations: 2-4; common. 


\section{Family: Naviculaceae}

65. Gyrosigma acuminata (Kütz.) Rab., Süssw.-Diat.: 47 (1853). [Syn.: Frustulia acuminata Kütz. (1832)]. Frustules 139-165 $\mu \mathrm{m}$ long, 23-25 $\mu \mathrm{m}$ broad. Stations: 1-4; abundant.

66. Gyrosigma attenuatum (Kütz.) Rab., Süssw.-Diat.: 47 (1853). [Syn.: Frustulia attenuate Kütz. (1833)]. Frustules 208-250 $\mu \mathrm{m}$ long, $25 \mu \mathrm{m}$ broad. Stations: 1-4; abundant.

67. Gyrosigma distortum (W. Smith) Cleve var. parkeri (M.B. Harrisson) Cleve, K. Svenska Vet.-Akad. Handl., ser. 4, 26(2): 116 (1894). Frustules 110-120 $\mu \mathrm{m}$ long, 16-17 $\mu \mathrm{m}$ broad. Stations: $1-4$, few.

68. Navicula cryptocephala Kütz., Bacillarien: 95 (1844). Frustules 25-35 $\mu \mathrm{m}$ long, 5-7 $\mu \mathrm{m}$ broad, 16 or 17 striae in $10 \mu \mathrm{m}$. Stations: 2-4; few. It is a new record for Bangladesh.

69. Navicula cuspidata Kütz., Bacillarien: 94 (1844). [Syn.: Frustulia cuspidata Kütz. (1833), N. fulva Ehr. (1838)]. Frustules 47-166 $\mu \mathrm{m}$ long, 16-32 $\mu \mathrm{m}$ broad, 11-18 striae in $10 \mu \mathrm{m}$. Stations: 1-4; common.

70. Navicula decussis Oestrup, DanSek Diat.: 77, pl. II, fig. 50 (1910). Frustules 16-25 $\mu \mathrm{m}$ long, 6-7 $\mu \mathrm{m}$ broad. Stations: 1-4; abundant. It is a new record for Bangladesh.

71. Navicula exigua (Dujardin) Nouv., Man. Obs. Micr. Atlas.: 44 (1842). Frustules 25$28 \mu \mathrm{m}$ long, $7.8 \mu \mathrm{m}$ broad. Stations: 1-4; abundant.

72. Navicula gastrum (Ehr.) Kütz., Bacillarien: 94 (1844). [Syn.: Pinnularia gastrum Ehr. (1843)]. Frustules 26-49 $\mu \mathrm{m}$ long, 7-10 $\mu \mathrm{m}$ broad. Stations: 2-4; few.

73. Navicula menisculus Schum., Schr. K. Phys.-Ökon. Ges. königs berg 8: 56 (1867). [Syn.: Navicula peregrima var. menisculus A. Mayer (1911)]. Frustules $28 \mu \mathrm{m}$ long, 6-7 $\mu \mathrm{m}$ broad. Stations: 1-4; abundant.

74. Navicula rhynchocephala Kütz., Bacillarien: 145 (1844). Frustules 50-65 $\mu \mathrm{m}$ long, 12-15 $\mu \mathrm{m}$ broad, 10 striae in $10 \mu \mathrm{m}$. Stations: $1,3,4$; few. It is a new record for Bangladesh.

75. Navicula viridula Kütz., Bacillarien: 91 (1844). Frustules 60-80 $\mu \mathrm{m}$ long, 13-15 $\mu \mathrm{m}$ broad, 6-9 striae in $10 \mu \mathrm{m}$. Stations: 1-4; few. It is a new record for Bangladesh.

76. Pinnularia acrosphaeria Bréb. var. laevis Cleve, K. Svenska Vet.-Akad. Handl., ser. 4, 27(3): 86 (1895). Frustules $83 \mu \mathrm{m}$ long, $12.7 \mu \mathrm{m}$ broad, 12-14 striae in $10 \mu \mathrm{m}$. Stations: $1-4$; abundant.

77. Pinnularia gibba var. mesogongyla (Ehr.) Hust., Pascher, Sussw.-fl. 10, 2. Aufl.: 327 (1930). [Syn.: P. mesogongyla Ehr. (1843)]. Frustules $44 \mu \mathrm{m}$ long, $10 \mu \mathrm{m}$ broad, 14 or 15 striae in $10 \mu \mathrm{m}$. Stations: 2, 3; few. 
78. Pinnularia gibba var. parva (Ehr.) Grun., An. Mus. Argent. Cienc. Nat. 37: 395 (1933). [Syn.: Navicula stauroptera var. parva Grunow (1860)]. Frustules 33-42 $\mu \mathrm{m}$ long, 8.3-9.0 $\mu \mathrm{m}$ broad, 9-12 striae in $10 \mu \mathrm{m}$. Stations: 2-4; few.

79. Pinnularia tabellaria Ehr., Abh. K. Akad.. Wiss. Berlin, Physik. K1. 1841: 422 (1843). Frustules $102 \mu \mathrm{m}$ long, $16 \mu \mathrm{m}$ broad (at the middle) and $11 \mu \mathrm{m}$ broad (at the tip), 15 striae in $10 \mu \mathrm{m}$. Stations: 3, 4; few.

80. Pleurosigma balticum var. simile (Ehr.) Grun., Ann. Mag Nat. Hist. ser. 2, 9: 8 (1852). Frustules $87 \mu \mathrm{m}$ long, $15 \mu \mathrm{m}$ broad, 19 or 20 striae in $10 \mu \mathrm{m}$. Stations: 1, 3; common.

81. Stauroneis anceps fa. gracilis (Ehr.) [Hustedt] Pascher, Süssw.-Fl. 10, 2, Aufl.: 256 (1930). Frustules $90-100 \mu \mathrm{m}$ long, $9-21 \mu \mathrm{m}$ broad, 20 or 25 striae in $10 \mu \mathrm{m}$. Stations: 2-4; common.

\section{Family: Cymbellaceae}

82. Cymbella affinis Kütz., Bacillarien: 80 (1844). [Syn.: Cocconema fusidium Ehr. (1838), Cymbella cymbiformis C. Agardh (1830)]. Frustules $83 \mu \mathrm{m}$ long, $18 \mu \mathrm{m}$ (at the middle), $11 \mu \mathrm{m}$ broad (at the tip). 13-15 striae in $10 \mu \mathrm{m}$. Station: 4; very rare.

83. Cymbella hustedtii Krasske, Bot. Arch. 3: 204, fig. 11 (1923). Frustules $36 \mu \mathrm{m}$ long, $11 \mu \mathrm{m}$ broad (at the middle), $7 \mu \mathrm{m}$ broad (at the tip), 13-15 striae in $10 \mu \mathrm{m}$. Station: 3; rare.

84. Cymbella parva (W. Smith) Kirchner, Alg. Schles.: 188 (1878). [Syn.: Cocconema parvum W. Smith (1853)]. Frustules $80 \mu \mathrm{m}$ long, $18 \mu \mathrm{m}$ broad (at the middle), $11 \mu \mathrm{m}$ broad (at the tip), 10-12 striae in $10 \mu \mathrm{m}$. Stations: 1, 3; very rare.

85. Cymbella stuxbergii (Cl.) Cleve, K. Sverska Vet.-Acad. Handl., ser. 4, 26(2): 174 (1894). [Syn.: Cocconema stuxbergii Cleve (1880)]. Frustules 50-69 $\mu \mathrm{m}$ long, 18-22 $\mu \mathrm{m}$ broad, 9-10 striae in $10 \mu \mathrm{m}$. Stations: 2-4; common.

86. Cymbella tumida (Bréb. ex Kütz.) Van Heurek, Syn. Diat. Belg. Expl., pl. 2, fig. 10 (1880). [Syn.: Cocconema tumidum Bréb. ex Kütz. (1849)]. Frustules 40-78 $\mu \mathrm{m}$ long, $12-20 \mu \mathrm{m}$ broad, $10-15$ striae in $10 \mu \mathrm{m}$. Stations: $2-4$; common.

87. Cymbella turgida Gregory, Quart. J. Micr. Sc. 4: 5 (1858). [Syn.: Cymbella eleginenia Krammer (1981)]. Frustules $56 \mu \mathrm{m}$ long, $15 \mu \mathrm{m}$ broad, 8 or 9 striae in 10 $\mu \mathrm{m}$. Station: 3; few.

88. Cymbella turgidula Grun., A. Schmidt, Atlas: pl. a, figs 23-29 (1875). Frustules 38$39 \mu \mathrm{m}$ long, $12-14 \mu \mathrm{m}$ broad, 9 or 10 striae in $10 \mu \mathrm{m}$. Stations: $1-4$; few.

89. Gomphonema augur Ehr., Ber. K. Akad. Wiss. Berlin 1840: 211 (1840). [Syn.: G. ehrenbergii Farrutherum (1864)]. Frustules 20-22 $\mu \mathrm{m}$ long, $7 \mu \mathrm{m}$ broad. Stations: 23 ; few. 
90. Gomphonema lanceolatum var. turris (Ehr.) Hust., Arch. Hydrobiol. suppl. 14: 166 (1935). [Syn.: G. turris Ehr. (1842)]. Frustules 54-73 $\mu \mathrm{m}$ long, 12-15 $\mu \mathrm{m}$ broad, 9-12 striae in $10 \mu \mathrm{m}$. Stations: 1-4; abundant.

91. Gomphonema longiceps var. subclavata (Ehr.) Grun., in Hustedt Pascher, Süssw-Fl. 10. 2. Aufl.: 375 (1930). [Syn.: G. montanum var. subclavatum Grun. (1880)]. Frustules $62 \mu \mathrm{m}$ long, $11 \mu \mathrm{m}$ broad. Station: 3; few.

92. Gomphonema olivaceum (Hornemann) Kütz., Bacillarien: 85 (1844). Frustules 45 $48 \mu \mathrm{m}$ long, 8.5-9.0 $\mu \mathrm{m}$ broad, $8-10$ stirae in $10 \mu \mathrm{m}$. Stations: 1,2 , 4; few.

\section{Family: Epithemiaceae}

93. Rhopalodia gibba (Ehr.) Ö Müller, Bot. Jahrb. 22: 65 (1895). [Syn.: Epithemia gibba (Ehr.) Kütz. (1844), Navicula gibba Ehr. (1832)]. Frustules 80-109 $\mu \mathrm{m}$ long, 18.2$21.8 \mu \mathrm{m}$ broad (at the middle), $11.0-14.5 \mu \mathrm{m}$ broad (at the tip) having sometimes median constrictions. Stations: 2-4; common.

\section{Family: Bacillariaceae}

94. Nitzschia acicularis (Kütz.) W. Smith, Syn. Brit. Diat. I: 43 (1853). [Syn.: Synedra acicularis Kutz. (1844)]. Frustules 35-100 $\mu \mathrm{m}$ long, 3-5 $\mu \mathrm{m}$ broad, striae 16-20 in 10 $\mu \mathrm{m}$. Stations: 2-4; few.

95. Nitzschia hantzschiana Rabh. in Grunow, Verh. K. Zool.-Bot. Ges. Wien 12: 576 (1862). Frustules $87 \mu \mathrm{m}$ long, $10 \mu \mathrm{m}$ broad, 10 canals in $10 \mu \mathrm{m}$. Stations: 1-4; common.

96. Nitzschia intermedia Hantzsch, Grunow K. Svenska Vet.-Akad. Handl., Ser. 4, 17(2): 95 (1880). Frustules 40-130 $\mu \mathrm{m}$ long, 4-6 $\mu \mathrm{m}$ broad, 9-12 striae in $10 \mu \mathrm{m}$. Stations: 1, 3; few. It is a new record for Bangladesh.

97. Nitzschia longissima (Bréb.) Grunow, Verh. k. Zool.-Bot. Ges. Wien 12: 581 (1862). [Syn.: Ceratoneis longissima Bréb. (1849), Nitzschia birostrata W. Smith (1853)]. Frustules $33 \mu \mathrm{m}$ long, $4 \mu \mathrm{m}$ broad. Stations: 2, 4; few.

\section{Discussion}

The studied pond is a unique habitat because of the fact that the limnological parameters showed wide range. Here carbon dioxide was occasionally undetectable at Stations 1 and 3, biocarbonate alkalinity was also undetectable at different times in all the stations and anoxia was observed in all the stations (Begum, 2008). Among the different phytoplankton groups, highest richness was represented by Euglenophyceae (50 taxa), Chlorophyceae (48 taxa) (Begum, 2008), and Bacillariophyceae (47 taxa) followed by Cyanophyceae (17 taxa) (Begum, 2008). Chrysophyceae, Xanthophyceae, Cryptophyceae 
and Dinophyceae were each represented by single species. Similar observation was made earlier by Islam et al. (1991) in organically polluted pond. Dominance of Euglenophyceae in the studied pond is supported by the observations made by Islam et al. (1991) and Begum and Hossain (1993). Record of euglenoid bloom under oxygen deficient condition is in agreement with observations made by previous workers (Hickmen and Penn, 1977; Begum and Hossain, 1993). The comparatively lower representation of Cyanophyceae among the major groups is in agreement with observations made by Islam et al. (1991) and Begum and Hossain (1993).

\section{Acknowledgments}

The author is grateful to late National Professor A.K.M. Nurul Islam, Department of Botany, University of Dhaka, for identification of some euglenoids and diatoms, and also grateful to Md. Zahangir Hossain for the assistance during the collection of the materials. Thanks are due to two textile mill authorities to sample their aquatic habitats.

\section{References}

Aziz, A. and Ara, M. 2000. Diatom taxa from deepwater rice fields at Tangail, Bangladesh. Bangladesh J. Plant Taxon. 7(1): 7-13.

Aziz, A. and Islam, A.K.M. Nurul 1986. Lagoon Algae of St. Martin's Island, Bangladesh. Dhaka Univ. Stud. Part E. 1(1): 45-52.

Aziz, A. and Tanbir, M. 2003. Algal flora of some northern districts of Bangladesh. Bangladesh J. Plant Taxon. 10(1): 63-77.

Begum, Z.N.T. 2008. A taxonomic account on the phytoplankton of a pond receiving textile industrial effluents. Bangladesh J. Plant Taxon. 15(2): 129-139.

Begum, Z.N.T. and Hossain, M.Z. 1993. Physico-chemical aspects and phytoplankton of a pond receiving textile industrial effluents. Dhaka Univ. J. Biol. Sci. 2(1): 93-99.

Begum, Z.N.T., Tarafdar, S.A. and Hossain, M.Z. 1996. Impact of major and minor elements on the plankton community of a pond receiving textile industrial effluents. Bangladesh J. Bot. 25(1): 65-72.

Bold, H.C. and Wynne, M.J. 1985. Introduction to the Algae. Prentice-Hall, New Jersey, pp. 1-706.

Germain, H. 1981. Flore Des diatomées. Diatomophycées Société Nouvelle Des éditions Boubée, Paris, pp. $1-444$.

Hickmen, M. and Penn, I.D. 1977. The relationship between planktonic algae and bacteria in a small lake. Hydrobiologia 53(2-3): 213-219.

Islam, A.K.M. Nurul and Alfasane, M.A. 2004. Euglenophyceae from Barisal district, Bangladesh: III. Genus Trachelomonas Ehr. Bangladesh J. Plant Taxon. 11(2): 33-37.

Islam, A.K.M. Nurul and Aziz, A. 1977. Studies on the phytoplankton of the Karnaphuli river estuary. J. Bangladesh Acad. Sci. 1(2): 141-145.

Islam, A.K.M. Nurul and Aziz, A. 1979. Algal flora of Moheshkhali Island, Bangladesh. Dacca Univ. Stud. Part B. 27(2): 105-122.

Islam, A.K.M. Nurul and Chowdhury, A.R. 1979. Hydrobiological studies of Dhanmondi Lake, Dacca. II. Phytoplankton. J. Asiatic Soc. Bangladesh (Sci.) 5(2): 47-57. 
Islam, A.K.M. Nurul and Haroon, A.K.Y. 1975. Limnological studies of the river Buriganga II. Biological Aspect. Dacca Univ. Stud. Part B. 23(1): 25-44.

Islam, A.K.M. Nurul and Hossain, M. 1979. Preliminary studies on the algal flora of Bagerhat, Khulna. J. Asiatic Soc. Bangladesh (Sci.) 5(2): 47-57.

Islam, A.K.M. Nurul and Irfanullah, H.Md. 2005. Hydrobiological Studies within the ten gardens at Srimangal, Bangladesh. II. Algal Flora (Excluding Chlorophyceae). Bangladesh J. Plant Taxon. 12(1): 33-52.

Islam, A.K.M. Nurul and Khatun, M. 1966. Preliminary studies on the phytoplanktons of polluted waters. Scientific Researches, East Regional Laboratories, Pakistan, 3(2): 94-109.

Islam, A.K.M. Nurul and Mannan, M.A. 1986. Algal flora of some brackish water shrimp culture ponds at Satkhira. Dhaka Univ. Stud. Part E. 1(1): 7-18.

Islam, A.K.M. Nurul and Moniruzzaman, K. 1981. Contribution to the study on Euglenophyta of Bangladesh. I. Genus Trachelomonas Ehr. Int. Revue ges. Hydrobiol. 66(1): 109-125.

Islam, A.K.M. Nurul, Khondker, M. and Haque, S. 1991. Euglenoid algae of four polluted ponds in and around Dhaka city. Bangladesh J. Bot. 20(1): 7-15.

Khondker, M., Islam, A.K.M. Nurul, Begum, Z.N.T. and Haque, S. 1990. Limnological studies of four polluted ponds in and around Dhaka city with reference to indicator species. Bangladesh J. Bot. 19(1): 51-63.

Nahar, K. 2001. Relationships between diatom assemblage of surface sediment and some environmental factors in two wetland ecosystems of Bangladesh. PhD Thesis, Department of Botany, University of Dhaka, pp. 1-257.

Round, F.E. 1985. The Ecology of Algae. Cambridge Univ. Press, Cambridge, pp. 1-653.

(Manuscript received on 21 January 2009; revised on 11 February 2009) 